\title{
Avaliação radiográfica de cães com displasia coxofemoral tratados pela sinfisiodese púbica
}

[Radiographic evaluation in dogs with hip dysplasia treated by pubic symphysiodesis]

\author{
L.A. Santana ${ }^{1}$, S.C. Rahal ${ }^{2}$, C.A. Estanislau ${ }^{2}$, S.E.R.S. Lorena ${ }^{3}$, V.M.V. Machado ${ }^{2}$, \\ D.P. Doiche $e^{3}$, O.C.M. Pereira-Júnior ${ }^{3}$ \\ ${ }^{1}$ Aluno de graduação - FMVZ-UNESP - Botucatu, SP \\ ${ }^{2}$ Faculdade de Medicina Veterinária e Zootecnia - UNESP Botucatu \\ Distrito de Rubião Júnior s/n \\ 18618-000 - Botucatu, SP \\ ${ }^{3}$ Aluno de pós-graduação - FMVZ- UNESP - Botucatu, SP
}

\begin{abstract}
RESUMO
Avaliou-se a progressão dos sinais radiográficos de cães com displasia coxofemoral ou potencialmente displásicos tratados pela técnica da sinfisiodese púbica. Foram utilizados 14 cães, seis machos e oito fêmeas, com idades entre quatro e seis meses. Após exame físico, procedeu-se ao exame radiográfico para avaliação da articulação coxofemoral, segundo padrão estabelecido pelo Colégio Brasileiro de Radiologia Veterinária. Três deles foram também avaliados pelo método PennHIP. Pelo histórico clínico, nove (64\%) animais foram trazidos por manifestarem sinais de dificuldade locomotora com os membros pélvicos, manifestada especialmente por claudicação unilateral ou bilateral e dificuldade de se levantar. Cinco $(36 \%)$ cães não tinham qualquer sinal clínico, e em quatro $(29 \%)$ realizou-se o exame radiográfico como prevenção. O sinal físico mais evidente foi dor à palpação da articulação coxofemoral, e dois (14\%) cães apresentaram sinal de Ortolani positivo. Com exceção de um cão que apresentou infecção nos pontos cutâneos, todos os demais evoluíram sem complicações no período pós-operatório. Radiograficamente houve progressão das lesões articulares em seis (43\%) animais e oito (57\%) mantiveram a classificação inicial. Concluiu-se que cães tratados pela sinfisiodese púbica não apresentam melhora dos sinais radiográficos iniciais.
\end{abstract}

Palavras-chave: cão, coxofemoral, sinfisiodese

\begin{abstract}
The radiographic findings in hip dysplastic or potentially hip dysplastic dogs treated by the pubic symphysiodesis were evaluated using 14 dogs - six males and eight females -, aging from four to six-monthold. After physical examination, the hip joint was evaluated by radiographic examination according to pattern established by the Colegio Brasileiro de Radiologia Veterinária. In addition, three dogs were evaluated by PennHIP method. According to medical history, nine (64\%) dogs were admitted due to signs of locomotor difficulty in pelvic limbs, especially characterized by uni or bilateral lameness and stiffness on rising. Five (36\%) dogs had no clinical signs. Radiographic examination was performed in four (29\%) dogs as prevention. The most evident physical sign was pain on manipulation of the hip with Ortolani-positive in two (14\%) dogs. Except for one dog that presented infection signs in the skin suture, no other postoperative complication was observed. The follow-up radiographic examination revealed progression of the degenerative articular changes in six (43\%) dogs, and eight (54\%) had no progression. In conclusion, the dogs treated by pubic symphysiodesis did not have improvement of the preoperative radiographic signs.
\end{abstract}

Keywords: dog, coxofemoral, symphysiodesis

Recebido em 20 de fevereiro de 2009

Aceito em 30 de agosto de 2010

*Autor para correspondência (corresponding author)

Email: sheilacr@fmvz.unesp.br 


\section{INTRODUÇÃO}

A displasia coxofemoral é o desenvolvimento anormal da articulação coxofemoral, o qual se inicia por uma frouxidão e progride para uma doença articular degenerativa (Haan et al., 1993; Arnbjerg, 1999). A etiologia da doença é multifatorial, porém são considerados fatores importantes a genética, o ambiente e a nutrição (Haan et al., 1993; Arnbjerg, 1999; Todhunter e Lust, 2003; Carneiro et al., 2006). A maioria dos cães afetados pertence às raças de porte médio a gigante (Haan et al., 1993; Todhunter e Lust, 2003). O diagnóstico clínico é baseado em anamnese, sinais clínicos, avaliação do modo de andar e palpação da articulação coxofemoral (Arnbjerg, 1999; Schulz, 2007). Contudo, o diagnóstico definitivo é feito por métodos de imagem, tais como radiografia pélvica ventrodorsal, como recomendado pelo Colégio Brasileiro de Radiologia Veterinária (CBRV) ou pela "Orthopedic Foundation for Animals" (OFA), radiografia em distração segundo o método PennHIP, medida da subluxação dorsolateral da cabeça femoral e métodos tomográficos (Haan et al., 1993; Swainson et al., 2000; Lust et al., 2001; Patricelli et al., 2002; Rocha e Tôrres, 2007; Normas ..., 2009), entre outros.

O tratamento é dependente da idade, da intensidade da lesão, do desempenho esperado do paciente e da presença de outras afecções, porém as metas incluem o alívio dos sinais clínicos da dor, a melhora da função e da qualidade de vida (Arnbjerg, 1999; Dassler, 2003; Schulz, 2007). O tratamento médico-conservativo consiste da utilização de anti-inflamatórios não esteroidais e analgésicos, para o controle da dor; administração de agentes condroprotetores; controle do peso por meio de dietas, exercícios e modificações de comportamento, com o intuito de diminuir o estresse na articulação (Haan et al., 1993; Remedios, 1995; Dassler, 2003). Entre os tratamentos cirúrgicos, podem ser citados a miectomia do músculo pectíneo, a osteotomia intertrocantérica, a osteotomia tripla da pélvis, a sinfisiodese púbica, a denervação articular, a substituição articular por prótese e a excisão da cabeça e colo femorais (Remedios, 1995; Arnbjerg, 1999; Schulz e Dejardin 2003; Schulz, 2007).

A sinfisiodese púbica é uma modalidade de tratamento considerada pouco invasiva, tecnicamente de fácil realização e indicada especialmente para cães jovens como um procedimento profilático (Dueland et al., 2001; Patricelli et al., 2002; Schulz e Dejardin, 2003; Manley et al., 2007). O método baseia-se no fechamento da porção púbica da sínfise pélvica, sem afetar o crescimento das suas porções isquiática e acetabular (Dueland et al., 2001; Patricelli et al., 2002; Manley et al., 2007). O procedimento tem sido efetuado por meio de destruição térmica da sínfise púbica ou sua excisão cirúrgica, seguida de aplicação de grampos (Swainson et al., 2000; Schulz e Dejardin, 2003). O propósito é aumentar a ventroversão acetabular na tentativa de diminuir a frouxidão articular (Mathews et al., 1996; Dueland et al., 2001; Schulz e Dejardin, 2003).

A idade da realização do procedimento parece refletir diretamente nos resultados (Schulz e Dejardin, 2003; Manley et al., 2007). Em estudo com cães displásicos, foi observado que a máxima rotação do ângulo acetabular ocorreu com a sinfisiodese púbica efetuada na idade de quatro meses, e a mínima com 24 meses de idade (Dueland et al., 2001). Adicionalmente, uma melhora significante na conformação e na frouxidão da articulação coxofemoral foi detectada em cães displásicos operados com a idade de 15 semanas quando comparados aos com 20 semanas (Patricelli et al., 2002).

Considerando-se o fato de a efetividade da sinfisiodese púbica ser ainda controversa, o presente trabalho teve por objetivo avaliar a progressão dos sinais radiográficos de cães displásicos ou potencialmente displásicos tratados pela técnica.

\section{MATERIAL E MÉTODOS}

Foram utilizados 14 cães de porte médio a gigante, seis machos e oito fêmeas, com idade entre quatro e seis meses, portadores ou potenciais portadores de displasia coxofemoral (Tab. 1).

Após exame clínico, todos os cães foram submetidos ao exame radiográfico para avaliação da articulação coxofemoral, segundo padrão estabelecido pelo CBRV. Três deles foram também avaliados pelo método PennHIP. O protocolo anestésico adotado foi $0,02 \mathrm{mg} / \mathrm{kg}$ de acepromazina $0,2 \%$ com $0,5 \mathrm{mg} / \mathrm{kg}$ de morfina pela via intramuscular, complementado 15 minutos após por $2 \mathrm{mg} / \mathrm{kg}$ de cloridrato de cetamina com $0,4 \mathrm{mg} / \mathrm{kg}$ de diazepam, via intravenosa. 
Tabela 1. Identificação do cão (raça, idade, sexo e peso corpóreo) no momento da realização da sinfisiodese, classificação radiográfica da displasia coxofemoral de acordo com padrão do CBRV, no período pré-operatório e na última avaliação pós-operatória (meses), e progressão dos sinais radiográficos

\begin{tabular}{|c|c|c|c|}
\hline Identificação & $\begin{array}{l}\text { Classificação } \\
\text { pré-operatória }\end{array}$ & $\begin{array}{l}\text { Classificação pós- } \\
\text { operatória }\end{array}$ & $\begin{array}{l}\text { Progressão } \\
\text { radiográfica }\end{array}$ \\
\hline $\begin{array}{c}\text { Dogue alemão, } 5,5 \text { meses, fêmea, } 28 \mathrm{~kg} \\
\text { (caso 1) }\end{array}$ & $\begin{array}{l}\text { MPD: C } \\
\text { MPE: B }\end{array}$ & $\begin{array}{l}\text { MPD: D } \\
\text { MPE: C } \\
\text { (18 meses) }\end{array}$ & Piora \\
\hline $\begin{array}{c}\text { Australian cattle dog, fêmea, } 4 \text { meses, } 10 \mathrm{~kg} \\
\text { (caso 2) }\end{array}$ & B & $\begin{array}{c}\text { B } \\
(13 \text { meses })\end{array}$ & Estável \\
\hline $\begin{array}{c}\text { Pastor alemão, } 5 \text { meses, macho, } 27 \mathrm{~kg} \\
\text { (caso 3) }\end{array}$ & $\mathrm{E}$ & $\begin{array}{c}\mathrm{E} \\
(13 \text { meses })\end{array}$ & Estável \\
\hline $\begin{array}{r}\text { Akita, } 4 \text { meses, fêmea, } 19 \mathrm{~kg} \\
\text { (caso } 4 \text { ) }\end{array}$ & B & $\begin{array}{c}\mathrm{B} \\
(13 \text { meses })\end{array}$ & Estável \\
\hline $\begin{array}{c}\text { Rottweiler, } 6 \text { meses, macho, } 35,2 \mathrm{~kg} \\
\text { (caso 5) }\end{array}$ & $\mathrm{E}$ & $\begin{array}{c}\mathrm{E} \\
(21 \text { meses })\end{array}$ & Piora \\
\hline $\begin{array}{c}\text { Sem raça definida, } 6 \text { meses, macho, } 40 \mathrm{~kg} \\
\text { (caso 6) }\end{array}$ & $\begin{array}{l}\text { MPD: C } \\
\text { MPE: B }\end{array}$ & $\begin{array}{c}\text { MPD: C } \\
\text { MPE: B } \\
\text { (21 meses) }\end{array}$ & Estável \\
\hline $\begin{array}{r}\text { Rottweiler, } 6 \text { meses, fêmea, 20,8kg } \\
\text { (caso 7) }\end{array}$ & $\begin{array}{l}\text { MPD: D } \\
\text { MPE: C }\end{array}$ & $\begin{array}{l}\text { MPD: E } \\
\text { MPE: D } \\
\text { (11 meses) }\end{array}$ & Piora \\
\hline $\begin{array}{c}\text { Bernese mountain dog, } 5 \text { meses, fêmea, } 21 \mathrm{~kg} \\
\text { (caso 8) }\end{array}$ & B & $\begin{array}{c}\mathrm{B} \\
(11 \text { meses })\end{array}$ & Estável \\
\hline $\begin{array}{l}\text { Samoieda, } 4 \text { meses, macho, } 14 \mathrm{~kg} \\
\text { (caso 9) }\end{array}$ & $\begin{array}{l}\text { MPD: B } \\
\text { MPE: C }\end{array}$ & $\begin{array}{l}\text { MPD: B } \\
\text { MPE: B } \\
\text { (18 meses) }\end{array}$ & Estável \\
\hline $\begin{array}{l}\text { Sem raça definida, } 5,5 \text { meses, macho, } 10 \mathrm{~kg} \\
\text { (caso 10) }\end{array}$ & A & $\begin{array}{c}\mathrm{B} \\
(10 \text { meses })\end{array}$ & Piora \\
\hline $\begin{array}{l}\text { Pastor alemão, } 5 \text { meses, fêmea, 14,4kg } \\
\text { (caso 11) }\end{array}$ & $\mathrm{E}$ & $\begin{array}{c}\mathrm{E} \\
(10 \text { meses })\end{array}$ & Estável \\
\hline $\begin{array}{c}\text { Sem raça definida, } 5 \text { meses, fêmea, } 16 \mathrm{~kg} \\
\text { (caso 12) }\end{array}$ & B & $\begin{array}{c}\text { B } \\
(39 \text { meses })\end{array}$ & Estável \\
\hline $\begin{array}{r}\text { Rottweiler, } 5 \text { meses, fêmea, } 32,5 \mathrm{~kg} \\
\text { (caso 13) }\end{array}$ & $\mathrm{E}$ & $\begin{array}{c}\mathrm{E} \\
(21 \text { meses })\end{array}$ & Piora \\
\hline $\begin{array}{c}\text { Labrador, 4,5 meses, macho, } 19 \mathrm{~kg} \\
\text { (caso } 14 \text { ) }\end{array}$ & $\mathrm{E}$ & $\begin{array}{c}\mathrm{E} \\
(19 \text { meses })\end{array}$ & Piora \\
\hline
\end{tabular}

CBVR: Colégio Brasileiro de Radiologia Veterinária.

A: sem sinais de displasia coxofemoral; B: articulações coxofemorais próximas do normal; C: displasia coxofemoral leve; D: displasia coxofemoral moderada; E: displasia coxofemoral severa.

Para a realização da sinfisiodese púbica, empregou-se a anestesia geral inalatória com isofluorane. Após a tricotomia das regiões abdominal caudal e púbica, os cães foram posicionados em decúbito dorsal. A antissepsia da área operatória foi com digluconato de clorexidina e, logo após, foram dispostos os panos de campos operatórios. Nas fêmeas, efetuou-se uma incisão de pele na linha média ventral estendendo-se do limite cranial do púbis até a sínfise pélvica cranial. Para os cães machos, a incisão de pele foi paralela ao pênis e estendeuse do púbis à sínfise pélvica cranial. O tecido subcutâneo foi incisado, seguido pela secção dos tendões pré-púbico e subpélvico. Os músculos grácil e adutor foram afastados da sínfise púbica (correspondente à metade cranial da sínfise pélvica), permanecendo a sínfise isquiática íntegra. Um protetor de borracha (cabo de gancho de castração) foi inserido dentro do canal pélvico e, ato contínuo, realizou-se a eletrocauterização da sínfise púbica por meio de eletrodo monopolar com ponta em espátula, em potência de $40 \mathrm{~W}$ por 10-15 segundos, com distâncias entre 2 e $3 \mathrm{~mm}$. Os músculos foram aproximados com pontos isolados em Sultan, o tecido subcutâneo com sutura contínua simples e a pele em pontos isolados simples. O fio utilizado foi o mononáilon 2-0 ou 3-0.

No pós-operatório, foram prescritos cefalexina, $30 \mathrm{mg} / \mathrm{kg}$ por via oral, a cada 12 horas, por cinco 
dias, e meloxicam, na dose de $0,1 \mathrm{mg} / \mathrm{kg}$ por via oral, a cada 24 horas, por até cinco dias. O tratamento da ferida cirúrgica foi com solução salina $0,9 \%$ e aplicação tópica de digluconato de clorexidina. Os pontos cutâneos foram removidos entre sete e 10 dias de pós-operatório.

Os cães foram radiografados, de acordo com a disponibilidade do proprietário, em períodos que variaram de 10 a 39 meses. Empregou-se o padrão estabelecido pelo CBRV para avaliação da articulação coxofemoral, com o mesmo protocolo anestésico previamente descrito. A evolução clínica foi também analisada. Em todos os casos, os proprietários foram advertidos sobre a necessidade da esterilização dos cães que, quando consentida, foi realizada a partir dos 10 meses de idade.

\section{RESULTADOS}

Pelo histórico clínico, nove $(64 \%)$ dos animais foram trazidos por manifestarem sinais de dificuldade locomotora com os membros pélvicos, manifestada especialmente por claudicação unilateral ou bilateral e dificuldade de se levantar. Apenas cinco (36\%) cães não tinham qualquer sinal clínico, e em quatro (29\%) realizou-se o exame radiográfico como prevenção. O sinal físico mais evidente foi dor à palpação da articulação coxofemoral, e dois (14\%) cães apresentaram sinal de Ortolani positivo. Entre os que foram submetidos ao PennHip (casos 1, 4 e 12), os valores do índice de distração estavam $\geq 0,50$.

Exceto um cão que apresentou sinais de infecção nos pontos cutâneos, todos os demais evoluíram sem complicações no período pós-operatório. Os proprietários relataram melhora dos sinais clínicos após o tratamento cirúrgico, com exceção de um caso. Pelo exame físico, três (21\%) dos animais apresentaram sinal de Ortolani positivo após a realização do procedimento. Radiograficamente houve progressão das lesões articulares em seis (43\%) dos animais, sendo que três deles ainda permaneceram na mesma classificação. Os demais mantiveram a classificação radiográfica inicial (Tab. 1) (Fig. 1). A conformação pélvica não sugeriu sinais de estreitamento em nenhum dos casos.

A esterilização foi efetuada em oito cães, seja por ovariosalpingo-histerectomia, orquiectomia ou vasectomia.

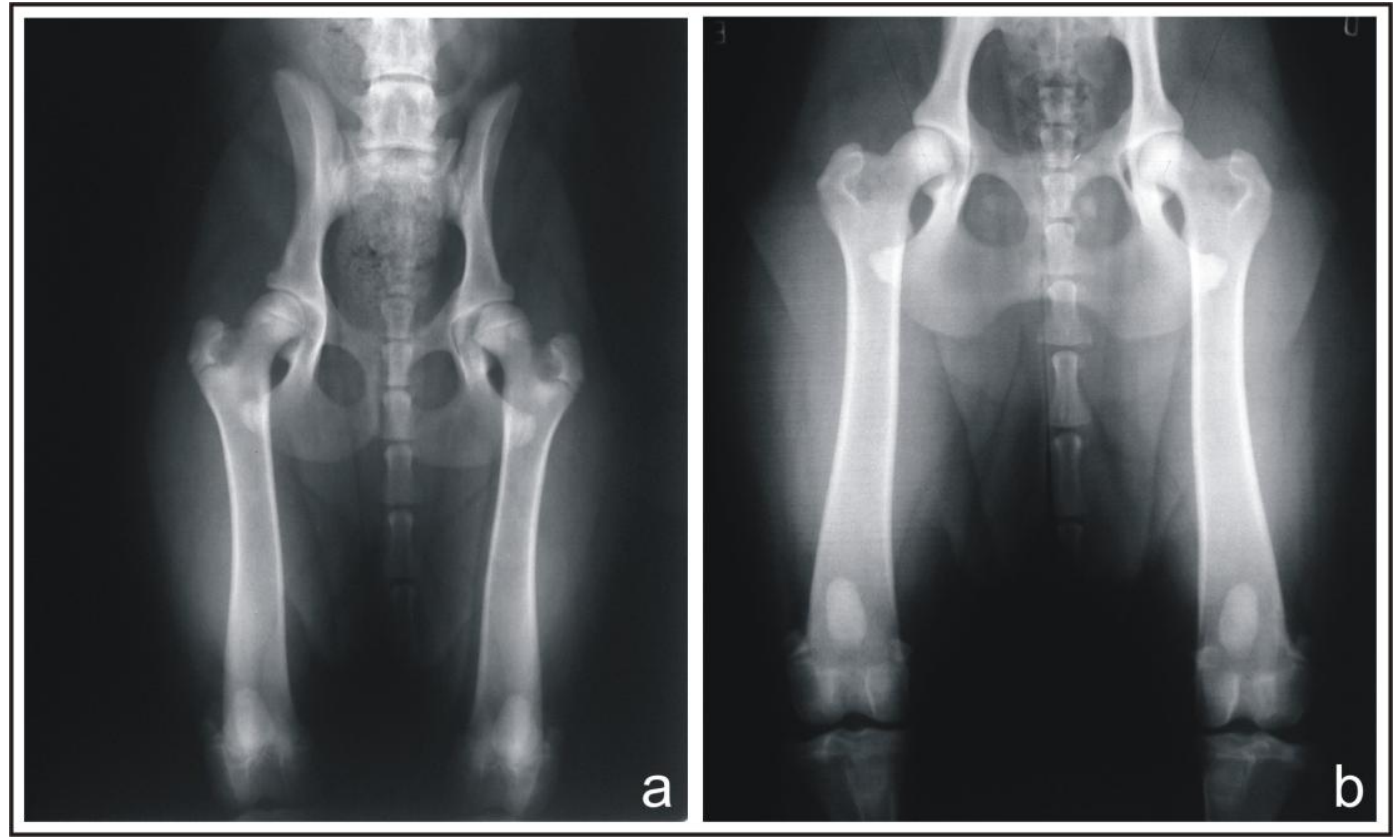

Figura 1. Aspecto radiográfico em posição ventrodorsal do cão Bernese mountain dog (caso 8), no préoperatório, aos cinco meses de idade (a) e 11 meses após o procedimento cirúrgico de sinfisiodese púbica (b). Observa-se a permanência na classificação B, de acordo com o padrão do Colégio Brasileiro de Radiologia Veterinária. 


\section{DISCUSSÃO}

Optou-se pela indução do fechamento da sínfise púbica por meio de cauterização, que, além de fácil realização, já teve sua efetividade comprovada (Dueland et al., 2001; Patricelli et al., 2001; 2002). Além disso, o uso de grampos, em estudo experimental com cães, mostrou-se inadequado, devido à migração desses um mês após o procedimento cirúrgico (Swainson et al., 2000).

De acordo com Schulz e Dejardin (2003), a cauterização pode ser efetuada na potência de 40W com eletrodo de agulha por até 30 segundos ou por eletrodo de espátula por até 10 segundos. Em estudo clínico com cães displásicos, foi usado eletrodo de agulha na dose de $40 \mathrm{~W}$ por 12 segundos (Patricelli et al., 2002) ou por 10-13 segundos (Manley et al., 2007). No presente relato, empregou-se o eletrocautério com ponta em espátula com algumas variações de tempo, ou seja, de 10 até 15 segundos, sendo o maior tempo utilizado em cães de porte grande e idade mais avançada. Como indicado por Patricelli et al. (2001), ao escolher a dosagem, deve-se levar em consideração a amplitude da placa fisária, de acordo com o indivíduo.

A palpação transretal é indicada durante a eletrocauterização, com o objetivo de mover a uretra e o reto da sínfise e assim protegê-los de lesões iatrogênicas (Patricelli et al., 2001; Schulz e Dejardin, 2003). Uma alternativa é o uso de retrator de borracha dentro do canal pélvico (Schulz e Dejardin, 2003). Essa última técnica foi adotada no presente estudo, porém com o emprego do cabo de gancho plástico de ováriohisterectomia, que mostrou ser efetivo e de baixo custo.

Embora seja indicada a ovariosalpingohisterectomia ou a orquiectomia dos animais acometidos conjuntamente com o procedimento de sinfisiodese púbica, com o intuito de evitar a perpetuação da displasia coxofemoral ou mesmo prevenir uma possível distocia decorrente de estreitamento pélvico em fêmeas (Swainson et al., 2000; Dueland et al., 2001; Schulz e Dejardin, 2003), no presente estudo optou-se pela realização desses procedimentos a partir dos 10 meses de idade. Como a gonadectomia prépuberal tem sido associada ao atraso do fechamento da placa fisária (Salmeri et al., 1991), sua influência sobre o desenvolvimento pélvico seria minimizada a partir desse período, uma vez que nos cães a sínfise púbica funde entre nove e 10 meses (Smith, 1964). Além disso, alguns proprietários foram refratários à orquiectomia, sendo, então, realizada nesses casos a vasectomia.

Segundo os autores, a rotação acetabular ventrolateral sobre a cabeça femoral induzida pelo fechamento da placa de crescimento púbica melhoraria a conformação da articulação coxofemoral (Mathews et al., 1996; Dueland et al., 2001). Considerando-se que o método radiográfico padronizado tanto pelo CBRV como pela OFA permite avaliar a conformação da articulação coxofemoral, assim como a presença de osteoartrite (Lust et al., 2001; Normas ..., 2009), foi possível observar que, no presente estudo, em seis (43\%) casos foi detectada progressão da doença osteoartrítica. Salienta-se ainda que avaliações com esses métodos diagnósticos antes dos dois anos de idade são consideradas preliminares, porque a subluxação pode não ser evidente, assim como os sinais de osteoartrite (Lust et al., 2001; Normas ..., 2009).

Em estudo clínico com cães displásicos com 15 $(n=6)$ e 20 semanas de idade $(n=6)$, não foram detectadas diferenças significativas no ângulo de Norberg e $25 \%$ deles desenvolveram doença osteoartrítica após a sinfisiodese púbica (Patricelli et al., 2002). Em estudo comparativo entre a osteotomia tripla da pélvis $(\mathrm{n}=9)$ e a sinfisiodese púbica ( $\mathrm{n}=9$ ), Manley et al. (2007) verificaram que ambas as técnicas não foram adequadas em prevenir a progressão da doença degenerativa em cães. Apesar dos achados radiográficos, no presente estudo, 13 (93\%) proprietários referiram-se à melhora da condição clínica ou à ausência de sinais após o tratamento. Como citado por Lust et al. (2001), apenas a evidência radiográfica de osteoartrite não assegura que os sinais clínicos de claudicação serão observados.

Uma das limitações da sinfisiodese púbica é a necessidade de um diagnóstico precoce, idealmente com quatro meses ou menos de idade, o que requer o emprego de métodos de distração como o PennHIP (Dueland et al., 2001; Patricelli et al., 2002; Schulz e Dejardin, 2003; Manley et al., 2007). Dos casos analisados, apenas quatro (29\%) foram trazidos ao serviço para diagnóstico preventivo. Contudo, a maioria dos cães possuía 
lesões displásicas classificadas entre as categorias B e E, de acordo com os critérios estabelecidos pelo CBRV. Vale fazer referência ao fato de que, ao empregarem a técnica em cães displásicos, Manley et al. (2007) selecionaram apenas os com índice de distração $\geq 0,50$ e com nenhuma, média ou moderada evidência de doença degenerativa.

Dessa forma, o potencial de sucesso da técnica é altamente dependente da idade do paciente e da severidade da lesão no momento da intervenção cirúrgica (Dueland et al., 2001; Manley et al., 2007), fato constatado no grupo de animais avaliados no presente estudo, haja vista a progressão das lesões nos cães com maior idade. Um fato questionável é se a técnica contribuiu para a menor progressão da lesão ou não, ou seja, se não operados, os cães teriam evoluído mais rapidamente para o processo degenerativo. Segundo Dueland et al. (2001), os resultados sugerem que cães com 24 meses ou mais velhos não seriam beneficiados por essa técnica, porque o crescimento pélvico e a remodelação acetabular seriam limitados.

Vale citar que a redução das dimensões pélvicas observada em cães após o emprego da sinfisiodese púbica não foi considerada de significância clínica (Swainson et al., 2000; Dueland et al., 2001). Embora não seja possível no presente estudo quantificar essa redução, visto não haver grupo-controle, o aspecto radiográfico não sugeriu sinais de estreitamento pélvico.

\section{CONCLUSÕES}

Os cães tratados pela sinfisiodese púbica apresentam progressão da doença articular degenerativa ou manutenção dos sinais radiográficos iniciais, isto é, não há melhora dos sinais iniciais da doença.

\section{REFERÊNCIAS BIBLIOGRÁFICAS}

ARNBJERG, J. Recent information about hip dysplasia. Vet. Clin N. Am.: Small Anim. Pract., v.29, p.921-934, 1999.

CARNEIRO, S.C.M.C.; FERREIRA, R.P.; FIORAVANTI, M.C.S. et al. Superalimentação e desenvolvimento do esqueleto de cães da raça Dogue Alemão: aspectos clínicos e radiográficos. Arq. Bras. Med. Vet. Zootec., v.58, p.511-517, 2006.
DASSLER, C.L. Canine hip dysplasia: diagnosis and nonsurgical treatment. In: SLATTER, D. (Ed). Textbook of small animal surgery. 3.ed. Philadelphia: Saunders, 2003. p.2019-2028.

DUELAND, R.T.; ADAMS, W.M.; FIALKOWSKI, J.P. et al. Effect of pubic symphysiodesis in dysplastic puppies. Vet. Surg., v.30, p.201-217, 2001.

HAAN, J.J.; BEALE, B.S.; PARKER, R.B. Diagnosis and treatment of canine hip dysplasia. Can. Pract., v.18, p.24-28, 1993.

LUST, G.; TODHUNTER, R.J.; ERB, H.N. et al. Comparison of three radiographic methods for diagnosis of hip dysplasia in eight-month-old dogs. J. Am. Vet. Med. Assoc., v.219, p.12421246, 2001.

MATHEWS, K.G.; STOVER, S.M.; KASS, P.H. Effect of pubic symphysiodesis on acetabular rotation and pelvic development in guinea pigs. Am. J. Vet. Res., v.57, p.1427-1433, 1996.

MANLEY, P.A.; ADAMS, W.M.; DANIELSON, K.C. et al. Long-term outcome of juvenile pubic symphysiodesis and triple pelvic osteotomy in dogs with hip dysplasia. J. Am. Vet. Med. Assoc., 230, p.206-210, 2007.

\section{NORMAS DO COLÉGIO BRASILEIRO DE RADIOLOGIA VETERINÁRIA. São Paulo: CBVR, 2009. Disponível em: <http://www.abrv.com.br/col_normas.php>. Acessado em: 27 jan. 2009.}

PATRICELLI, A.J.; DUELAND, R.T.; LU, Y. et al. Canine pubic symphysiodesis: investigation of electrocautery dose response by histologic examination and temperature measurement. Vet. Surg., v.30, p.261-268, 2001.

PATRICELLI, A.J.; DUELAND, R.T.; ADAMS, W.M. et al. Juvenile pubic symphysiodesis in dysplastic puppies at 15 and 20 weeks of age. Vet. Surg., v.31, p.435-444, 2002.

REMEDIOS, A.M. Treatment of canine hip dysplasia: a review. Can. Vet. J., v.36, p.503509, 1995.

ROCHA, B.D.; TÔRRES, R.C.S. Ultrasonic and radiographic study of laxity in hip joints of young dogs. Arq. Bras. Med. Vet. Zootec., v.59, p.90-96, 2007. 
SALMERI, K.R.; BLOOMBERG, M.S.; SCRUGGS, S.L. et al. Gonadectomy in immature dogs: effects on skeletal, physical, and behavioral development. J. Am. Vet. Med. Assoc., v.198, p.1193-1203, 1991.

SCHULZ, K.S. Diseases of the joints. In: FOSSUM, T.W. (Ed). Small Animal Surgery. St. Louis: Mosby Elsevier, 2007. p.1143-1315.

SCHULZ, K.S.; DEJARDIN, L.M. Surgical treatment of canine hip dysplasia. In: SLATTER, D. (Ed). Textbook of small animal surgery. 3.ed. Philadelphia: Saunders, 2003. p.2029-2059.
SMITH, R.N. The pelvis of the young dog. Vet. Rec., v.76, p.975-979, 1964.

SWAINSON, S.W.; CONZEMIUS, M.G.; RIEDESEL, E.A. et al. Effect of pubic symphysiodesis on pelvic development in the skeletally immature Greyhound. Vet. Surg., v.29, p.178-190, 2000.

TODHUNTER, R.J.; LUST, G. Hip dysplasia: pathogenesis. In: SLATTER, D. (Ed). Textbook of small animal surgery. 3.ed. Philadelphia: Saunders, 2003. p.2009-2019. 